\title{
Vieses Cognitivos entre Consumidores num Dilema Social Simulado: Falso Consenso, Erro Fundamental de Atribuição, de Ator-Observador e de Autosserviço
}

\author{
Fabio Iglesias ${ }^{1}$ \\ Víthor Rosa Franco \\ Júlia Villela Teixeira Gisler \\ Douglas Leite Piasson \\ Universidade de Brasilia
}

\begin{abstract}
RESUMO - Decisões entre cooperar e competir são fundamentais em dilemas sociais, quando interesses individuais de consumo estão em conflito com benefícios coletivos. O objetivo desta pesquisa foi testar a ocorrência de vieses de cognição social em um dilema simulado, com base nos procedimentos de Gifford e Hine (1997). Após jogarem o software FISH em condições de cooperação e competição, os participantes relataram suas auto e hetero percepções. Foram verificados o viés de falso consenso e o erro fundamental de atribuição, mas os vieses de autosserviço e o ator-observador se mostraram efeitos mais limitados e apenas entre cooperadores. Discutem-se algumas implicações teóricas, metodológicas e práticas dos fenômenos no contexto do consumo sustentável.
\end{abstract}

Palavras-chave: dilemas sociais, vieses cognitivos, cooperação

\section{Cognitive Biases Among Consumers in a Simulated Social Dilemma: False- Consensus, Fundamental Attribution Error, Actor-Observer, and Self-Serving Asymmetry}

\begin{abstract}
Decisions between cooperating and competing are central to social dilemmas, that is, when individual needs are at odds with collective benefits. The aim of this paper was to test the occurrence of social cognition biases in a simulated social dilemma, by using Gifford and Hine (1997) procedures. After taking part in FISH, a micro-world simulation, either in conditions of cooperation or competition, participants reported their self- and other perceptions. False consensus and fundamental error of attribution were found, while self-serving and actor-observer biases showed more limited effects and only among cooperators. Some theoretical, methodological, and practical implications are discussed in the context of sustainable consumption.
\end{abstract}

Keywords: social dilemmas, cognitive biases,; cooperation

Parte significativa das atividades de consumo envolvem dilemas sociais, caracterizados por conflitos entre interesses individuais e interesses coletivos (Dawes, 1980; Van Lange, Joireman, Parks, \& Van Dijk, 2013). Os consumidores têm à sua disposição uma quantidade geralmente finita de recursos (produtos, serviços, pessoas, espaço), que devem ser de alguma maneira compartilhados. Ao se comportar de modo mais individualista, visando à acumulação de recursos, adotam inevitavelmente uma perspectiva mais competitiva. Por outro lado, ao se comportar de um modo mais coletivista, abrindo mão do consumo excessivo ou dividindo recursos com outras pessoas, adotam inevitavelmente uma perspectiva mais cooperativa. No entanto, decidir entre cooperar ou competir em dilemas não depende só de processos objetivos, sofrendo também influência direta da percepção que as pessoas têm umas sobre as outras, visto que essas percepções podem ativar associações inconscientes de ações comportamentais apropriadas nessas situações (Kelley, Holmes, Kerr, Reis, Rusbult, \& Van Lange, 2003).

1 Endereço para correspondência: ICC Sul Sala A1-116, Laboratório de Psicologia Social, Instituto de Psicologia, Campus Universitário Darcy Ribeiro, Asa Norte, Brasília, DF, Brasil. CEP: 70.900-100. E-mail: fabiglesias@gmail.com
Tomando como base o procedimento experimental de Gifford e Hine (1997), o objetivo desta pesquisa foi testar a ocorrência de quatro vieses cognitivos entre consumidores num dilema social simulado: o viés de falso consenso (Ross, Greene, \& House, 1977); o erro fundamental de atribuição (Heider, 1958; Jones \& Davis, 1965), também chamado de viés de correspondência (Gilbert \& Malone, 1995); o viés ator-observador (Jones \& Nisbett, 1972); e o viés de autosserviço (Miller \& Ross, 1975), constituindo o que a literatura de psicologia social experimental tem classificado como replicação conceitual com variações metodológicas (Brandt et al., 2014).

\section{Dilemas Sociais e o Consumo de Recursos Escassos}

Questões que envolvem recursos ambientais têm sido as mais tipicamente utilizadas para descrever a lógica do dilema social nos comportamentos de consumo, considerando-se frequentemente a importância de variáveis culturais, como por exemplo diferenças em relação à independência ou interdependência do self (Kopelman, 2009). No Brasil as ameaças ao fornecimento e distribuição de energia elétrica 
ocorridas nos anos de 2001 e 2002, que deram origem à chamada crise do apagão, servem como um exemplo típico. Segundo Rosa (2001), devido à diminuição de chuvas naquele período e à grande dependência brasileira da energia hidroelétrica, o país se viu em riscos de blecaute, o que exigiu uma drástica redução do consumo em quase todas as regiões geográficas. Isso motivou uma série de ações governamentais que incluíram racionamentos forçados, cortes nos gastos e concessão de benefícios (ou aplicação de punições) para atingir metas de consumo. Quanto ao mercado, observou-se um estímulo ainda hoje valorizado de se adquirir aparelhos elétricos e eletrônicos que consumam menos energia, lâmpadas mais econômicas e implementações arquitetônicas que possam tornar os ambientes de trabalho e residenciais mais "inteligentes" nesse critério.

Todo esse cenário foi acompanhado por uma ampla campanha pública para a mudança de comportamento, mas como reage um consumidor quando confrontado com a informação de que deve economizar energia em sua residência ou no trabalho? Como o dilema social é por ele percebido, ainda que intuitivamente? Diante do apelo para que, por exemplo, reduza o tempo em seu banho quente diário com o uso de chuveiro elétrico, o consumidor se vê numa situação complexa que pode, ainda assim, ser simplificada em duas opções de comportamento: a) cooperar com a dinâmica do racionamento, reduzindo seu tempo de banho (perda individual) visando a um impacto positivo no consumo global de energia (ganho coletivo); b) não cooperar, mantendo o seu consumo (ganho individual), que por sua vez ocasionará em um impacto negativo no consumo global (perda coletiva).

Alguns fatores ajudam a potencializar a decisão por comportamentos cooperativos em dilemas sociais, tanto individuais como situacionais (Alencar, Siqueira, \& Yamamoto, 2008). Entre os mais importantes, destacamse o número de pessoas envolvidas (quanto maior, menor a probabilidade de haver cooperação), a necessidade de se estabelecerem metas superordenadas que exigem a colaboração de todos, sanções para prevenir a atuação de competidores que explorem os cooperadores, assim como discutir e estabelecer normas mutuamente compartilhadas (Kopelman, Weber, \& Messick, 2002). Uma meta-análise conduzida por Balliet (2010) revelou que a comunicação em dilemas sociais apresenta altos tamanhos de efeito no aumento da cooperação, mesmo quando se trata de um fenômeno de larga escala. Parte do problema, portanto, envolve como o consumidor percebe seus próprios comportamentos e os comportamentos das outras pessoas envolvidas: "Será que elas também vão colaborar?"; "Quais suas motivações?"; "Devo cuidar das minhas necessidades individuais ou devo correr o risco de estar me sacrificando enquanto os outros continuam consumindo?".

\section{Cooperação e Competição em Dilemas Sociais Simulados}

Dilemas sociais podem ser definidos por três características principais: as decisões competitivas sempre geram melhores resultados para uma pessoa em particular (independentemente do que as outras fazem); sempre geram algum tipo de prejuízo às outras; e esse prejuízo é sempre maior do que o ganho pessoal dos envolvidos (Van Lange, Liebrand, Messick, \& Wilke, 1992). A sustentabilidade de qualquer sistema, portanto, exige a promoção de comportamentos mais cooperativos do que competitivos, mesmo que estes últimos pareçam mais convenientes ou atraentes para os consumidores envolvidos. Por essa relação com as consequências do comportamento ser fundamental para entender o próprio comportamento e os fenômenos de cognição social que possam influenciar essas percepções, a necessidade de maior validade externa é uma preocupação em pesquisas de dilemas sociais. É fundamental investigálos quando variáveis situacionais relevantes estão em jogo e os participantes não estão somente relatando atitudes em questionários, mas exibindo padrões mais comportamentais.

Por sua relação com a presente pesquisa, merece destaque um dilema de escassez de recursos concebido por Edney (1979) e nomeado como jogo das nozes. A situação, criada em laboratório, oferece a dois ou mais jogadores a oportunidade de retirar de uma tigela com uma determinada quantidade de nozes (ou fichas, balas, etc), quantas eles quiserem. A regra mais importante que o experimentador estabelece é que, depois da escolha feita por cada jogador, o número de nozes que restarem na tigela será duplicado. $\mathrm{O}$ jogo continua até que o experimentador decida encerrá-lo ou até que não restem mais nozes na tigela, por não poderem mais ser duplicadas. Esse procedimento se configura como um micromundo do que acontece em situações reais que dependem da sustentabilidade dos recursos disponíveis. Se cada pessoa consumir apenas uma fração adequada e sempre restar uma quantidade razoável do recurso (p.ex. árvores), ele eventualmente se recupera e o consumo pode continuar indefinidamente.

Desenvolvido preliminarmente por Gifford e Wells (1991) e em sua versão mais aprimorada por Gifford e Gifford (2000), o software FISH 3.1 simula um dilema de escassez com uma tarefa de pesca para se investigar comportamentos de gestão de recursos finitos. Instalado em qualquer computador pessoal, ele permite ao pesquisador o controle de diversas variáveis, relativas ao comportamento dos pescadores virtuais e aos ganhos e perdas relativos à pesca. $\mathrm{O}$ participante pode jogar contra o computador ou pode "pescar" conectado em rede com outros participantes do mesmo jogo, sendo essa uma das variáveis manipuláveis. A dinâmica do software se assemelha ao jogo das nozes de Edney (1979), porque oferece a um ou mais jogadores a oportunidade de consumir a quantidade de recursos que quiserem, até que se chegue na temporada que o experimentador programou como a final ou quando não sobrarem mais recursos a serem consumidos.

O emprego do FISH para a análise de dilemas sociais é apoiado no relato de evidências de validade interna e externa por seus autores. Apesar do aspecto lúdico, apresenta uma situação simulada que é comparável a uma situação concreta. As atividades de pesca representam, tanto a nível global quanto local, algo que é suficientemente familiar a quaisquer participantes em potencial. Mesmo que não se envolvam diretamente com a pesca na vida real, a maior parte das pessoas pode facilmente conceber que, se realizada em demasia, pode diminuir consideravelmente a disponibilidade 
e a reprodução de peixes (ou mesmo esgotá-los). Por sua relevância prática, muitos estudos têm usado cenários dessa natureza para investigar dilemas, além de frequentemente usados como caso de ensino em ecologia, sociologia, economia e psicologia (Wentworth \& Ratté, 2002).

\section{Quatro Vieses Cognitivos}

Utilizando o jogo das nozes, Gifford e Hine (1997) testaram a ocorrência de cinco vieses relevantes de julgamento na área de cognição social e que são investigados em diversos contextos, por sua relação direta com os fenômenos de atribuição de causalidade. O valor desses fenômenos reside nas discrepâncias sistemáticas entre o julgamento dos próprios comportamentos e o julgamento dos comportamentos e atitudes percebidos nos outros. Quatro desses vieses foram abordados na presente pesquisa: o efeito de falso consenso, o erro fundamental de atribuição, o efeito ator-observador e o viés de autosserviço. Não foi aqui incluído o viés autocentrado, quinto fenômeno investigado por aqueles autores, porque a literatura da área tem mostrado que carece de clareza conceitual para se distinguir do viés de autosserviço, como sumarizaram Fiske e Taylor (2013).

$\mathrm{O}$ efeito do falso consenso é definido como a tendência de acreditar que as outras pessoas agem da mesma forma que nós, dada uma mesma situação (Ross, Greene, \& House, 1977). Isso pode envolver escolhas atitudinais ou comportamentais, sendo um fenômeno sistematicamente investigado na percepção de normas sociais, nos estudos sobre intenções de votos e sobre orientação política de modo geral. Mais recentemente, tem sido também investigado no comportamento virtual online (Wojcieszak, 2011) e na atitude frente a celebridades (Bui, 2012). Ele costuma ser operacionalizado pela percepção de que altas porcentagens da população expressam o mesmo posicionamento do ator, que acredita representar o que é normal ou comum.

Crisp e Turner (2010) argumentam que o viés de falso consenso tem como base uma heurística de disponibilidade, já que nossas crenças e opiniões pessoais são facilmente recuperadas na memória, sendo portanto muito mais acessíveis em tarefas de estimação social. Mesmo que as pessoas não acreditem que a ampla maioria das outras possuam o mesmo posicionamento, no viés de falso consenso elas acabam superestimando essa real porcentagem (Krueger \& Clement, 1997).

O segundo viés cognitivo aqui pesquisado é o erro fundamental de atribuição (Ross, 1977), como ficou mais popularizado o viés de correspondência, identificado primeiramente por Jones e Davis (1965). Ao julgar o comportamento de outros, tipicamente valoriza-se mais a influência de fatores internos, tais como a personalidade e outras características individuais do ator observado. $\mathrm{O}$ fenômeno tem suas bases no trabalho seminal de Heider (1958) sobre atribuição de causalidade e constitui um dos temas mais populares dentro e fora da psicologia social. A rigor, existe um debate sobre diferenças e semelhanças entre o erro fundamental de atribuição e o viés de correspondência (Gawronski, 2004; Gilbert \& Malone, 1995). Para os objetivos deste trabalho, no entanto, a análise conceitual do problema não interfere na sua análise empírica e os fenômenos são aqui tratados como sinônimos. De forma sumária, o erro fundamental de atribuição é operacionalizado pela superestimação de fatores disposicionais em detrimento de fatores situacionais, quando estes últimos podem explicar mais acuradamente o comportamento observado. Ele pode ocorrer tanto no nível individual quanto grupal. Allison e Kerr (1994) mostraram, por exemplo, que em dilemas sociais os grupos com resultados percebidos como satisfatórios são vistos como mais cooperativos e competentes (características internas) do que grupos com resultados não satisfatórios.

No viés ator-observador (Jones \& Nisbett, 1972), o ator do comportamento tende a considerar que os fatores externos são os mais importantes para determinar a causalidade de seu próprio comportamento, enquanto o observador atribui a causalidade a fatores internos do ator. É, portanto, um fenômeno relacionado ao erro fundamental de atribuição. Algumas situações são tipicamente utilizadas para descrever esse viés, como nos estudos sobre pedidos de desculpas (Saraiva \& Iglesias, 2013). Para explicar o comportamento de atraso de seu colega de trabalho, por exemplo, um indivíduo pode recorrer a características disposicionais, como na conclusão de que ele se atrasou por ser descompromissado com horário, desatento ou irresponsável, entre outros. Quando o atraso ocorre com ele mesmo, no entanto, provavelmente recorre a atribuições situacionais, como o trânsito engarrafado, a um problema inesperado no relógio despertador ou à culpa de terceiros.

Finalmente, no viés de autosserviço, o que está em jogo é a consequência do comportamento observado, seja ele próprio ou de outras pessoas. Quando bem sucedido, o ator tende a atribuir a causalidade de modo mais interno, ou seja, identificando algum traço ou característica sua que pareça explicar o sucesso. Quando mal sucedido, no entanto, o ator tende a recorrer a fatores e situações externas (Miller \& Ross, 1975). O viés parece cumprir uma função de preservar a autoestima e de regular reações emocionais, algo que já tem sido investigado por meio de técnicas de mapeamento cerebral (Blackwood, Bentall, Fytche, Simmons, Murray, \& Howard, 2003). Ainda mais notório, verifica-se que com frequência as pessoas atribuem a si mesmas a causa de eventos que nem poderiam ter qualquer relação factível com seu desempenho individual (Campbell \& Sedikides, 1999). Embora não seja aqui objeto de investigação, o fenômeno tem também a sua contrapartida: as pessoas tendem a negar a responsabilidade por algo que tenha saído errado (Fiske \& Taylor, 2013).

Considerando esses aportes teóricos e metodológicos, a presente pesquisa testou a ocorrência dos quatro vieses cognitivos entre jogadores num dilema social simulado. Já que dilemas sociais no FISH podem ser configurados de modo mais cooperativo ou competitivo, essas duas condições experimentais foram aqui utilizadas como fatores de interação na manifestação dos vieses, que podem favorecer a cooperação ou a competição dos participantes. Foram elaboradas, portanto, quatro hipóteses.

A primeira hipótese prevê efeito de falso consenso, assim como interação com a condição experimental e a forma como o participante decidiu se comportar (cooperativa ou competitivamente). Já a segunda hipótese prevê erro 
fundamental de atribuição, assim como interação com a condição experimental. Em seguida, a terceira hipótese prevê efeito ator-observador, assim como interação com a condição experimental, com a forma como o participante decidiu se comportar (cooperativa ou competitivamente) e com a forma que o participante julga ter se comportado (cooperativa ou competitivamente). Finalmente, a quarta hipótese prevê uma correlação positiva entre a satisfação do participante com o resultado do jogo e a relevância dada ao próprio comportamento para esse resultado, assim como uma interação com a condição experimental e com a forma que o participante decidiu se comportar (cooperativa ou competitivamente).

\section{Método}

\section{Participantes}

Participaram do experimento 101 estudantes universitários (55,4\% homens), com média de idade de 19,93 anos (DP $=2,69)$. Eles foram recrutados diretamente em locais de circulação de pessoas numa instituição de ensino superior e alocados aleatoriamente em duas condições experimentais: cooperativa $(n=50)$ e competitiva $(n=51)$. Não foram consideradas outras variáveis de diferenciação sociodemográfica da amostra, dada a relativa homogeneidade dessas características em alunos universitários, tampouco foram consideradas as áreas de seus cursos de origem.

\section{Materiais e Instrumentos}

Os materiais utilizados incluíram o software FISH 3.1 (Gifford \& Gifford, 2000), cujo conteúdo em inglês foi traduzido para o português e retrotraduzido, garantindo fidelidade às instruções para os participantes. Ele foi instalado em um notebook convencional, apoiado em mesa e banco portátil, para que a coleta não dependesse do ambiente de laboratório e os participantes pudessem ser abordados em corredores, salas de aula desocupadas e outros espaços de convivência. $\mathrm{O}$ instrumento para mensuração dos vieses cognitivos se apresentou na forma de um questionário, baseado em Gifford e Hine (1997), composto por oito itens medidos com escalas de 11 categorias (de 0 a 10), descritas na Tabela 1.

Para a criação das duas condições experimentais o jogo foi configurado pelo que é definido operacionalmente no FISH como nível de ganância (greediness). Trata-se de uma variável que altera a velocidade e o número de peixes que os jogadores virtuais estão programados a pescar, em uma escala de 0 (pouco ganancioso) a 1 (muito ganancioso). Conforme recomendações de Gifford e Gifford (2000), na condição experimental de cooperação um dos pescadores virtuais foi definido para apresentar um nível de ganância de 0,4 , enquanto na condição experimental de competição um dos pescadores apresentava um nível de 0,6. Os outros dois pescadores virtuais, em ambas as condições, foram programados para se comportar de forma mais "neutra", com base em um nível de ganância de 0,5 .

Foi também utilizado o índice IR (individual restraint), que indica a taxa de racionamento individual, calculado no FISH por meio das mesmas variáveis utilizadas para determinar o nível de ganância dos jogadores virtuais. A escala desse indicador varia entre 1 - $\mathrm{Ne}$ e , sendo $\mathrm{N}$ o número de pescadores no jogo. Números inferiores a 0 indicam competição e números acima de 0 indicam cooperação. Dessa forma, o nível de cooperação e competição dos participantes pode ser determinado tanto de maneira escalar quanto dicotômica.

\section{Procedimentos}

Os participantes foram abordados pessoalmente e convidados a participar da tarefa, apresentada como uma pesquisa sobre gestão de recursos. Embora tenha caráter

Tabela 1. Vieses Cognitivos, Operacionalização dos Itens e Escalas Utilizadas para Mensurá-los.

\begin{tabular}{|c|c|c|}
\hline Viés & Operacionalizações dos itens e variáveis & Escala \\
\hline \multirow[b]{2}{*}{ Falso consenso } & Índice IR (individual restraint) no FISH. & Dicotômica \\
\hline & $\begin{array}{l}\text { "Suponha que todos os alunos desta universidade participassem do estudo. Qual porcentagem } \\
\text { representa o número de alunos que escolheria maximizar seus próprios ganhos?" }\end{array}$ & 0 a 100 \\
\hline \multirow{2}{*}{$\begin{array}{l}\text { Erro fundamental } \\
\text { de atribuição }\end{array}$} & $\begin{array}{l}\text { "Você considera que as características pessoais (traços de personalidade, atitudes) dos outros } \\
\text { pescadores são relevantes para explicar a maneira como eles agiram durante o jogo?" }\end{array}$ & 0 a 10 \\
\hline & $\begin{array}{l}\text { "Você considera que as características situacionais (quantidade de peixes, comportamento dos } \\
\text { outros pescadores) são relevantes para explicar a maneira como os outros jogadores agiram } \\
\text { durante o jogo?" }\end{array}$ & 0 a 10 \\
\hline \multirow{3}{*}{ Ator-observador } & $\begin{array}{l}\text { "Você considera que suas características pessoais foram relevantes para explicar como você agiu } \\
\text { durante o jogo? }\end{array}$ & 0 a 10 \\
\hline & $\begin{array}{l}\text { "Você considera que as características situacionais foram relevantes para explicar como você agiu } \\
\text { durante o jogo?" }\end{array}$ & 0 a 10 \\
\hline & "Durante o jogo você considera ter agido de maneira mais cooperativa ou mais competitiva?" & 0 a 10 \\
\hline \multirow{2}{*}{ Autosserviço } & "Quão relevante foi a sua atuação no resultado final do jogo?" & 0 a 10 \\
\hline & & 0 a 10 \\
\hline
\end{tabular}


claramente lúdico, destacou-se sua característica de simulação no computador, evitando no convite o uso de termos relacionados a cooperação, competição, compartilhamento ou divisão, que poderiam influenciar o padrão de comportamento dos participantes. Após o consentimento dos participantes e garantias de anonimato, o experimentador descrevia sumariamente a tarefa no computador e apresentava o questionário de vieses ao final. As instruções do dilema foram apresentadas na própria tela do computador, descrevendo as regras que são basicamente as mesmas do jogo das nozes de Edney (1979). Após o término da tarefa experimental no FISH e da aplicação do questionário, os participantes receberam esclarecimentos sobre todos os objetivos da pesquisa, atendendo às recomendações éticas previstas.

\section{Resultados}

A primeira hipótese testada foi a do viés de falsoconsenso. Inicialmente verificou-se que, independentemente da condição experimental, os participantes estimaram que $69 \%$ das outras pessoas se comportariam de maneira competitiva se expostas à mesma situação. Em seguida a amostra foi classificada entre os que agiram de modo cooperativo e os que agiram de modo competitivo, com base no IR (individual restraint) do FISH. Uma ANOVA fatorial 2 (comportamento cooperativo ou competitivo) x 2 (condição experimental cooperativa ou competitiva) mostrou que a estimativa feita pelos participantes sobre a porcentagem de alunos que escolheria maximizar seus próprios ganhos foi maior entre os que jogaram competitivamente $(M=75,81$, $D P=12,52)$ do que entre os que jogaram cooperativamente $(M=63,35, D P=21,01), F(1,96)=16,87, p<0,001, \eta_{P}{ }^{2}=$ 0,15 . No entanto, a proporção foi estimada como maior entre os jogadores alocados à condição experimental cooperativa $(M=70,87, D P=19,77)$ do que à condição competitiva $(M=$ $67,80, D P=17,79), F(1,96)=4,60, p=0,03, \eta_{P}{ }^{2}=0,05$. Não se verificou uma interação da classificação dos participantes pelo IR com a condição experimental, $F(1,96)<1, p=0,75$.

Para testar a ocorrência do erro fundamental de atribuição, segundo viés cognitivo hipotetizado, foram comparadas as médias das duas questões sobre o quanto as características disposicionais ou as situacionais explicam o comportamento dos outros jogadores. Uma MANOVA para medidas repetidas mostrou que, de modo geral, os participantes fizeram mais atribuições disposicionais $(M=7,50, D P=2,05)$ do que situacionais $(M=6,88, D P=2,57), F(1,98)=5,01, p=$ $0,03, \eta_{P}{ }^{2}=0,05$. Esses resultados não diferiram em função da condição experimental, $F(1,96)<1, p=0,44$.

Para o terceiro viés cognitivo, o do ator-observador, compararam-se as médias das questões sobre atribuição disposicional e sobre atribuição situacional do comportamento dos próprios jogadores. Uma MANOVA para medidas repetidas mostrou que essas duas autoatribuições não diferiram, mesmo quando se considerou a classificação de IR dos jogadores e as duas condições experimentais $F_{S}(1$, $98)<1, p s=n s$. Tampouco encontraram-se diferenças nessas médias quando se considerou o quanto os jogadores se acharam mais cooperativos ou mais competitivos, $F(1$, $88)<1, p=0,91$. Tomando-se apenas os jogadores que se comportaram cooperativamente segundo o critério IR $(n=52)$, a autoatribuição situacional foi maior que a disposicional, $t(51)=1,58, p=0,12, d=0,30$.

Finalmente, para a ocorrência do viés de autosserviço, testou-se a hipótese de que haveria uma correspondência entre a satisfação do participante com o resultado do jogo e a relevância dada ao próprio comportamento para esse resultado. Não se verificou, no entanto, correlação significativa entre essas variáveis, considerando-se toda a amostra, $r=0,15$, $p=0,14$. A mesma tendência foi observada quando essas variáveis foram isoladas por condição experimental, ps $=n s$. Já no caso da análise por padrão de comportamento conforme o critério IR, verificou-se que a correlação não foi significativa para os que agiram competitivamente, $r=0,06$, $p=0,70$, mas apresentou tendência de correlação positiva, ainda que marginal, para os que agiram cooperativamente, $r=0,23, p=0,09$.

\section{Discussão}

Este estudo teve por objetivo testar a ocorrência de quatro vieses cognitivos em um dilema social que simulou o consumo de recursos escassos, assim como o efeito das condições em que se manipularam experimentalmente um ambiente mais cooperativo ou mais competitivo e o efeito da estratégia de consumo adotada pelo participante. Num plano geral, verificou-se que os quatro vieses foram confirmados, mas em função de diferentes critérios analíticos. $O$ viés de falso-consenso e o erro fundamental de atribuição se mostraram robustos, ao se considerar a amostra como um todo. Entretanto, o viés ator-observador e o viés de autosserviço foram dependentes de como os participantes cooperaram ou competiram, a despeito da manipulação experimental.

Embora haja uma discussão conceitual sobre a lógica do fenômeno enquanto mecanismo de projeção social (Biernat \& Eidelman, 2007), operacionalmente o viés de falso-consenso é identificado na percepção de que a maior parte das outras pessoas pensaria ou agiria da mesma forma que nós, numa mesma situação. O dado inicial dessa variável mostrou que, em média, os participantes estimaram que mais de dois terços da população agiria de forma competitiva. Esse resultado foi maior para os que agiram competitivamente no dilema simulado, mas também se mostrou alto para os que agiram de maneira cooperativa. Notadamente, aqueles expostos à condição experimental de cooperação mostraram ainda maior viés de falso-consenso. Trata-se, portanto, de uma evidência clara de pessimismo ou desconfiança no contexto do consumo consciente, algo já identificado no Brasil por Cardoso e Souza (2013). Nota-se, sobretudo, como isso se expressa em situações de compartilhamento de recursos escassos, quando tipicamente as pessoas interagem com desconhecidos sobre os quais podem apenas fazer inferências. Como revisaram Goldstein e Cialdini (2009), percepções falsas de consenso podem influenciar diretamente a maneira como consumidores decidem, por exemplo, reutilizar suas toalhas em quartos de hotéis, economizar energia residencial ou reciclar o lixo, quando solicitadas a fazer isso com apelos pró-ambientais. 
A hipótese do erro fundamental de atribuição também foi confirmada, ainda que tenha revelado um tamanho de efeito pequeno. Assim como o viés de falso consenso, também envolve discussões sobre sua ocorrência (Miyamoto \& Kitayama, 2002), mas a polêmica sobre o quanto é apropriado descrevê-lo como um erro efetivamente "fundamental" está associada a resultados de algumas amostras asiáticas, que mostram maiores atribuições situacionais do que disposicionais. É, de qualquer maneira, um fenômeno amplamente verificado em psicologia social (cf. Fiske \& Taylor, 2013), que explica por que as pessoas tendem a fazer, de forma mais automática (e frequentemente menos acurada), atribuições internas ao comportamento de outros. Nesta pesquisa a ocorrência do erro fundamental de atribuição não dependeu da condição experimental, portanto ele pode ser observado tanto naqueles que são estimulados a cooperar quanto nos que são estimulados a competir em situações de consumo compartilhado. Por outro lado, alguns trabalhos têm mostrado as possibilidades de se reverter atribuições equivocadas, que minimizem a culpabilização das outras pessoas quando variáveis situacionais explicam melhor seus comportamentos (West, 1996). Trata-se de um caminho importante para intervenções da psicologia social aplicada a várias áreas que, assim como na escola, no tribunal e na redução do preconceito, possam ser implementadas nos problemas que envolvem consumo.

Ao atribuir causas ao próprio comportamento, as pessoas tendem a dar mais importância para variáveis situacionais, o que caracteriza o viés ator-observador, fenômeno que estende o erro fundamental de atribuição (Jones \& Nisbett, 1972). Entretanto, os dados aqui encontrados mostraram que apenas participantes que cooperaram realmente apresentaram esse viés. Apesar de não se ter verificado diferença pelo critério convencional, o tamanho de efeito indicou ainda assim uma tendência a maior atribuição situacional ao próprio comportamento entre os cooperadores. É possível que aqueles que cooperam percebam o ambiente do dilema social como mais hostil e potencialmente insustentável, julgando portanto que seu desempenho individual tem menor impacto e depende mais da ação de fatores não disposicionais. Essa é justamente a dinâmica que se quer evitar no gerenciamento de recursos escassos em dilemas sociais, buscando-se promover a percepção de que o próprio comportamento faz sim uma diferença considerável no sistema como um todo, ainda mais quando se soma o comportamento de vários consumidores. Robins, Spranca e Mendelsohn (1996) argumentam que o viés ator-observador pode depender de diferenças individuais de quem faz a atribuição e da história de exposição a situações similares e seus resultados. Muitos dilemas sociais como o aqui investigado têm essa característica de se repetirem sistematicamente na vida real, tais como racionar energia, água e outros recursos que podem se apresentar como escassos.

O viés de autosserviço se caracteriza como tomar para si maior responsabilidade quando se percebe que uma interação resultou em uma consequência satisfatória, mas negar a responsabilidade pessoal quando a consequência é percebida como ruim. Esse efeito foi aqui marginalmente significativo, mas apenas para os participantes que agiram de forma cooperativa. Vale considerar, no entanto, que na literatura essa hipótese é tipicamente avaliada via correlação, apesar de qualquer manipulação experimental envolvida, como mostra a meta-análise conduzida por Mezulis, Abramson, Hyde e Hankin (2004). Isso não somente limita as inferências sobre o tipo de relação das variáveis envolvidas, como também, pode ser muito sensível à distribuição dos dados. É razoável compreender, de qualquer forma, que as pessoas que mostram comportamentos mais competitivos (e mais negativos para o dilema social) não têm qualquer motivação para assumir a responsabilidade pelos resultados do consumo compartilhado, sejam eles satisfatórios ou insatisfatórios.

\section{Comentários Finais}

Ainda que não se qualifique nem tenha sido uma tentativa de replicação direta (Brandt et al., 2014), algumas considerações podem ser desenvolvidas quando se comparam os resultados aqui obtidos com aqueles encontrados por Gifford e Hine (1997), que inspiraram os procedimentos metodológicos e analíticos adotados. $\mathrm{O}$ estudo original relatou a confirmação de cinco vieses cognitivos hipotetizados, pois além de falso consenso, erro fundamental de atribuição, ator-observador e autosserviço, foi verificado também o viés autocentrado. No entanto, aqueles autores também reconheceram possíveis efeitos moderadores de consequências pessoais e coletivas nos padrões de consumo exibidas no dilema social. Embora possa ser uma explicação relevante para o fato de aqui não se ter verificado a ocorrência de todos os vieses na mesma maneira, obviamente outros fatores podem ter tido maior influência, como a natureza específica da tarefa, assim como o fato de que no estudo original os participantes podiam ser recompensados financeiramente. Mais central do que essa diferença, no entanto, pode ser o fato de que no presente estudo os participantes jogaram o FISH sozinhos, mesmo que se apresentasse uma situação de disputa de recursos com jogadores virtuais. Gifford e Hine coletaram os dados em situações de grupo, em que os participantes efetivamente interagiam, pelo menos visualmente, com os outros.

Embora aqueles autores não tenham relatado medidas de tamanho de efeito para seus testes $t$, elas foram aqui calculadas para permitir uma comparação com as ANOVAs utilizadas no presente estudo, com base na estratégia de conversão de Ruscio (2008). Nessa análise, por exemplo, o viés de falso consenso revelou um tamanho de efeito expressivamente superior, enquanto o erro fundamental de atribuição revelou um tamanho de efeito expressivamente inferior àqueles encontrados no estudo original. Ambos resultados podem ser entendidos à luz de diferenças culturais, associadas aos contextos em que as coletas de dados foram realizadas - Canadá e Brasil. Enquanto o primeiro país é caracterizado por altos escores de individualismo, o segundo tem um perfil mais coletivista (Hofstede, 2003). Morling e Masuda (2013) revisaram recentemente esses tipos de diferenças em cognição social, mostrando como se estendem para diversos vieses cognitivos além do erro fundamental de atribuição e do falso consenso. Poucos dados brasileiros foram produzidos sobre isso, no entanto, de modo que o presente estudo pode representar uma contribuição relevante tanto para questões básicas quanto aplicadas. 
Questões aplicadas no contexto do consumo de recursos escassos são muito evidentes. Dilemas sociais dependem fundamentalmente de escolhas mais cooperativas em detrimento de escolhas mais competitivas, para que haja consumo sustentável e atendimento de demandas individuais e coletivas (Capraro, 2013). Dadas as dificuldades metodológicas de se investigar experimentalmente uma série de dilemas sociais no mundo real, a simulação dessas situações em laboratório aparece como uma alternativa viável, com relativo controle de variáveis, muito menos sujeita a problemas de ordem ética e envolvendo baixos custos de implementação (Smithson \& Foddy, 1999). DiFonzo, Hantula e Bordia (1998) argumentam ainda que, no caso de simulações em computador que se apresentam como micromundos, é possível reunir evidências robustas não só de validade interna, mas também de validade externa, algo que tende a ser alvo dos críticos de pesquisas em laboratório na psicologia (Berkowitz \& Donnesrtein, 1982). Investigar como as pessoas percebem seus próprios comportamentos e os das outras pessoas envolvidas em tarefas dessa natureza pode ser paradigma valioso e generalizável para fenômenos de larga escala, que se expressam na forma de boicotes, consumismo, manifestações sociais, racionamentos e especulação imobiliária, entre outros problemas de consumo aos quais a psicologia social se aplica.

\section{Referências}

Alencar, A. I., Siqueira, J. O., \& Yamamoto, M. E. (2008). Does group size matter? Cheating and cooperation in Brazilian school children. Human Evolution and Behavior, 29, 42-48.

Allison, S.T., \& Kerr, N.L. (1994). Group correspondence biases and the provision of public goods. Journal of Personality and Social Psychology, 66, 688-698.

Balliet, D. (2010). Communication and cooperation in social dilemmas: A meta-analytic review. Journal of Conflict Resolution, 54, 39-57.

Berkowitz, L., \& Donnerstein, E. (1982). External validity is more than skin deep: Some answers to criticisms of laboratory experiments. American Psychologist, 37, 245-257.

Biernat, M., \& Eidelman, S. (2007). Standards. In A. W. Kruglanski and E. T. Higgins (Eds.), Social psychology: Handbook of basic principles (pp. 308-333). New York: Guilford.

Blackwood, N.J., Bentall, R.P., Fytche, D.H., Simmons, A., Murray, R.M., \& Howard, R.J. (2003). Self-responsibility and the selfserving bias: An fMRI investigation of causal attributions. Neuroimage, 20(2), 1076-1085.

Brandt, M. J., IJzerman, H., Dijksterhuis, A., Farach, F. J., Geller, J., Giner-Sorolla, R., Grange, J. A., Perugini, M., Spies, J. R., \& Veer, A. v. (2014). The replication recipe: What makes for a convincing replication? Journal of Experimental Social Psychology, 50, 217-224.

Bui, N.H. (2012). False consensus in attitudes toward celebrities. Psychology of Popular Media Culture, 1(4), 236-243.

Campbell, W. K., \& Sedikides, C. (1999). Self-threat magnifies the self-serving bias: A meta-analytic integration. Review of General Psychology, 3(1), 23-43.

Capraro, V. (2013). A model of human cooperation in social dilemmas. PLoS ONE, 8(8), e72427.
Cardoso, B. L., \& Souza, A. M. (2013). Consumo consciente e sua influência no comportamento do consumidor: Uma análise da recente publicação científica do Brasil. Trabalho apresentado no $\mathrm{IV}^{\circ}$ Colóquio Organizações, Desenvolvimento e Sustentabilidade, Belém, PA.

Crisp, R. J., \& Turner, R. N. (2010). Essential social psychology. London: Sage

Dawes, R. M. (1980). Social dilemmas. Annual Review of Psychology, 31, 169-193.

DiFonzo, N., Hantula, D. A., \& Bordia, P. (1998). Microworlds for experimental research: Having your (control and collection) cake, and realism too. Behavior Research Methods, Instruments, \& Computers, 30, 278-286.

Edney, J. J. (1979). The nuts game: A concise commons dilemma analog. Environmental Psychology and Nonverbal Behavior, $3,252-254$.

Fiske, S. T., \& Taylor, S. E. (2013). Social cognition: From brains to culture. New York: McGraw-Hill.

Gawronski, B. (2004). Theory-based bias correction in dispositional inference: The fundamental attribution error is dead, long live the correspondence bias. European Review of Social Psychology 15(1), 183-217.

Gifford, J., \& Gifford, R. (2000). FISH 3: A microworld for studying social dilemmas and resource management. Behavior Research Methods, Instrumentation, and Computers, 32, 417- 422.

Gifford, R., \& Hine, D. W. (1997). "I'm cooperative but you're greedy": Some cognitive tendencies in a commons dilemma. Canadian Journal of Behavioural Science, 29(4), 257-265.

Gifford, R., \& Wells, J. (1991). FISH: A commons dilemma simulation. Behavior Research Methods, Instruments and Computers, 23, 437-441.

Gilbert, D. T., \& Malone, P. S. (1995). The correspondence bias. Psychological Bulletin, 117, 21-38.

Goldstein, N. J., \& Cialdini, R. B. (2009). Normative influences on consumption and conservation behaviors. In M. Wänke, Social psychology of consumer behavior (pp. 273-296). New York: Psychology Press.

Hardin, G. (1968). The tragedy of the commons. Science, 162, 1243-1248.

Heider, F. (1958). The psychology of interpersonal relations. New York: Wiley.

Hofstede, G. (2003). Culture's consequences: Comparing values, behaviors, institutions, and organizations across nations. Thousand Oaks, CA: Sage.

Jones, E. E., \& Davis, K. E. (1965). From act to dispositions: The attribution process in person perception. Em L. Berkowitz (Ed.), Advances in experimental social psychology (Vol. 2, pp. 220-266). New York: Academic Press.

Jones, E. E., \& Nisbett, R. E. (1972). The actor and the observer: Divergent perceptions of the causes of behavior. Em E. E. Jones, D. E. Kanouse, H. H. Kelley, R. E. Nisbett, S. Valins, \& B. Weiner. (Eds.), Attribuition: Perceiving the causes of behavior (pp. 79-94). Morrisstown, NJ: General Learning.

Kelley, H. H., Holmes, J. G., Kerr, N. L., Reis, H. T., Rusbult, C. E., \& Van Lange, P. A. M. (2003). An atlas of interpersonal situations. New York: Cambridge University Press.

Kopelman, S. (2009). The effect of culture and power on cooperation in commons dilemmas: Implications for global resource management. Organization Behavior and Human Decision Processes, 108, 153-163. 
Kopelman, S., Weber, J. M., \& Messick, D. M. (2002). Factors influencing cooperation in commons dilemmas: A review of experimental psychological research. Em E. Ostrom, T. Dietz, N. Dolsak, P. C. Stern, S. Sonich \& E. U. Weber (Eds.), The drama of the commons (pp. 113-156). Washington: National Academy Press.

Krueger, J., \& Clement, R. W. (1997). Estimates of social consensus by majorities and minorities: The case for social projection. Personality and Social Psychology Review, 1(4), 299-313.

Mezulis, A. H., Abramson, L. Y., Hyde, J. S., \& Hankin, B. L. (2004). Is there a universal positivity bias in attributions? A meta-analytic review of individual, developmental, and cultural differences in the self-serving attributional bias. Psychological bulletin, 130(5), 711-747.

Miller, D. T., \& Ross, M. (1975). Self-serving biases in the attribution of causality: Fact or fiction? Psychological Bulletin, $82,213-225$.

Miyamoto, Y., \& Kitayama, S. (2002). Cultural variation in correspondence bias: The critical role of attitude diagnosticity. Journal of Personality and Social Psychology, 83, 1239-1248.

Morling, B., \& Masuda, T. (2012). Social cognition in real worlds: Cultural psychology and social cognition. In S.T. Fiske \& C. N. Macrae (Eds.) Handbook of social cognition (pp. 429-450). Los Angeles, CA: Sage.

Robins, R. W., Spranca, M. D., \& Mendelsohn, G. A. (1996). The actor-observer effect revisited: Effects of individual differences amd repeated social interactions on actor and observer atributtions. Journal of Personality and Social Psychology, 71(2), 375-389.

Rosa, L. P. (2001). O apagão: Por que veio? Como sair dele? Rio de Janeiro: Revan.
Ross, L. (1977). The intuitive psychologist and his shortcomings: Distortions in the attribution process. Em L. Berkowitz (Ed.), Advances in experimental social psychology (Vol. 10, pp. 173220). New York: Academic Press.

Ross, L., Greene, D., \& House, P. (1977). The false consensus phenomenon: An attributional bias in self-perception and social perception processes. Journal of Experimental Social Psychology, 13, 279-301.

Saraiva, R. B., \& Iglesias, F. (2013). Julgamentos de plausibilidade e reações emocionais a desculpas. Interação em Psicologia, 17, 163-170.

Smithson, M. J., \& Foddy, M. (1999). Theories and strategies for studying social dilemmas. Em M. Foddy, M. Smithson, S. Schneider \& M. Hogg (Eds.), Resolving social dilemmas: Dynamic, structural, and intergroup aspects (pp. 1-14). Philadelphia: Psychology Press.

Van Lange, P. A. M., Joireman, J., Parks, C. D., \& Van Dijk, E. (2013). The psychology of social dilemmas: A review. Organizational Behavior and Human Decision Processes, 120, 125-141.

Van Lange, P. A. M., Liebrand, W. B. G., Messick, D. M., \& Wilke, H. A. M. (1992). Social dilemmas: The state of the art. Em W. B. G. Liebrand, D. M. Messick \& H. A. M. Wilke (Eds.), A social psychological approach to social dilemmas (pp. 3-28). New York: Pergamon.

Wentworth, D. R., \& Ratté, K. (2002). Fish tales: Classroom lessons about the economics and the environment. Bozeman, MT: Property and Environment Research Center.

West, P. M. (1996). Predicting preferences: An examination of agent learning. Journal of Consumer Research, 23, 68-80.

Wojcieszak, M. (2011). Computer mediated false consensus: Radical online groups, social networks and news media. Mass Communication \& Society, 14, 527-546.

Recebido em 07.07.2014

Primeira decisão editorial em 02.11.2014

Versão final em 26.11.2014

Aceito em 15.03.2015 